\title{
Dielectric Haloscopes: A New Way to Detect Axion Dark Matter
}

\author{
Allen Caldwell, ${ }^{1}$ Gia Dvali, ${ }^{1,2,3}$ Béla Majorovits, ${ }^{1}$ Alexander Millar, ${ }^{1}$ Georg Raffelt, ${ }^{1}$ Javier Redondo, ${ }^{1,4}$ \\ Olaf Reimann, ${ }^{1}$ Frank Simon, ${ }^{1}$ and Frank Steffen ${ }^{1}$ \\ (MADMAX Working Group) \\ ${ }^{1}$ Max-Planck-Institut für Physik (Werner-Heisenberg-Institut), Föhringer Ring 6, 80805 München, Germany \\ ${ }^{2}$ Ludwig-Maximilians-Universität, Theresienstraße 37, 80333 München, Germany \\ ${ }^{3}$ CCPP, New York University, New York, New York 10003, USA \\ ${ }^{4}$ University of Zaragoza, Pedro Cerbuna 12, 50009 Zaragoza, Spain
}

(Received 23 November 2016; published 3 March 2017)

\begin{abstract}
We propose a new strategy to search for dark matter axions in the mass range of $40-400 \mu \mathrm{eV}$ by introducing dielectric haloscopes, which consist of dielectric disks placed in a magnetic field. The changing dielectric media cause discontinuities in the axion-induced electric field, leading to the generation of propagating electromagnetic waves to satisfy the continuity requirements at the interfaces. Large-area disks with adjustable distances boost the microwave signal $(10-100 \mathrm{GHz})$ to an observable level and allow one to scan over a broad axion mass range. A sensitivity to QCD axion models is conceivable with 80 disks of $1 \mathrm{~m}^{2}$ area contained in a $10 \mathrm{~T}$ field.
\end{abstract}

DOI: 10.1103/PhysRevLett.118.091801

Introduction.-The nature of dark matter (DM) is one of the most enduring cosmological mysteries. One prime candidate, the axion, arises from the Peccei-Quinn (PQ) solution to the strong $C P$ problem, the absence of $C P$ violation in quantum chromodynamics (QCD). The $C P$ violating QCD phase $\theta$ is effectively replaced by the axion field whose potential is minimal at $\theta=0$ [1-3]. Thus, $\theta$ dynamically relaxes towards zero regardless of its initial conditions, satisfying the neutron electric dipole moment constraints $\theta \lesssim 10^{-11}$ [4].

Tiny relic oscillations with a frequency given by the axion mass $m_{a}$ around $\theta=0$ persist, acting as cold DM [5-9]. If DM is purely axionic, its local galactic density $\rho_{a}=\left(f_{a} m_{a}\right)^{2} \theta_{0}^{2} / 2 \sim 300 \mathrm{MeV} / \mathrm{cm}^{3}$ implies that $\theta \sim$ $\theta_{0} \cos \left(m_{a} t\right)$ at Earth, with $\theta_{0} \sim 4 \times 10^{-19}$. While these oscillations could be detected, the main challenge is to scan over a huge frequency range, as $m_{a}$ is unknown.

However, cosmology can guide our search. Causality implies that, at some early time, $\theta$ is uncorrelated between patches of the causal horizon size. We consider two cosmological scenarios depending on whether cosmic inflation happens after (A) or before (B) that time.

In scenario $\mathrm{A}$, one patch is inflated to encompass our observable Universe while smoothing $\theta$ to a single initial value $\theta_{I}$. The cosmic axion abundance depends on both $\theta_{I}$ and $m_{a}$, so the DM density can be matched for any $m_{a}$ allowed by astrophysical bounds [10] for a suitable $\theta_{I}$.

In scenario $\mathrm{B}$, the axion abundance is given by the average over random initial conditions and the decay of accompanying cosmic strings and domain walls. Freed from the uncertainty in the initial conditions, scenario B provides a concrete prediction $m_{a} \sim 100 \mu \mathrm{eV}[11,12]$, although with some theoretical uncertainty [13].

Searches based on cavity resonators in strong magnetic fields (Sikivie's haloscopes [14]) such as ADMX [15], ADMX HF [16], and CULTASK [17] are optimal for $m_{a} \lesssim 10 \mu \mathrm{eV}$. Much lower values of $m_{a}$ can be explored by nuclear magnetic resonance techniques like CASPER [18] or with $L C$ circuits $[19,20]$.

The mass range favored in scenario $B$ is untouched by current experiments, and for cavity haloscopes will remain so for the foreseeable future. While fifth-force experiments [21] could search this region, they would not directly reveal the nature of DM. We present here a new concept to cover this important gap, capable of discovering $\sim 100 \mu \mathrm{eV}$ mass axions. It consists of a series of parallel dielectric disks with a mirror on one side, all within a magnetic field parallel to the surfaces, as shown in Fig. 1-a dielectric haloscope.

For a large $m_{a}$ the greatest hindrance for conventional haloscopes is that the signal is proportional to the cavity volume $V$. With dimensions on the order of the axion Compton wavelength $\lambda_{a}=2 \pi / m_{a}, V \propto \lambda_{a}^{3}$, which decreases rapidly with $m_{a}$. (We use natural units with $\hbar=c=1$ and the Lorentz-Heaviside convention $\alpha=e^{2} / 4 \pi$.) While there are plans to couple multiple high-quality cavities, use open resonators, or compensate with extremely high magnetic fields and/or new detectors, these techniques may not prove practical for large $m_{a}$ 's [17,22-24].

A radical approach for increasing the volume is to use a dish antenna (i.e., a mirror) inside a $B$ field to convert axion DM into microwaves [25]. The resonantly enhanced 


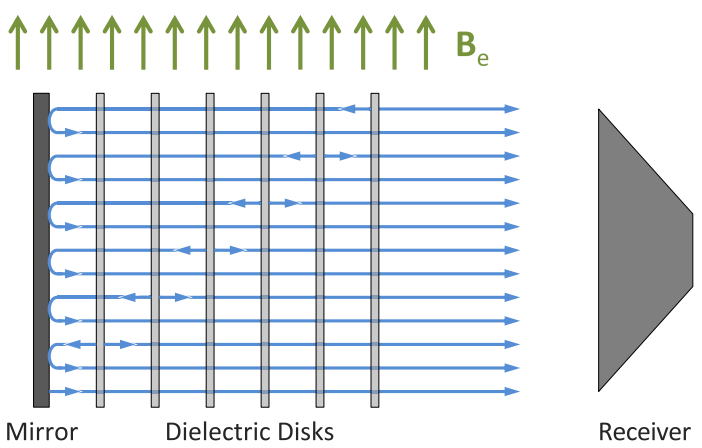

FIG. 1. A dielectric haloscope consisting of a mirror and several dielectric disks placed in an external magnetic field $\mathbf{B}_{e}$ and a receiver in the field-free region. A parabolic mirror (not shown) could be used to concentrate the emitted power into the receiver. Internal reflections are not shown.

axion-photon conversion is then given up in favor of a large transverse area where $A \gg \lambda_{a}^{2}$.

In our dielectric haloscope, each disk emits electromagnetic (EM) waves similar to the mirror case. As the disks are semitransparent, the waves emitted by each disk are reflected by and transmitted through the other disks before exiting. With suitable disk placement, these waves add coherently to the emitted power considerably with respect to a single mirror. While the use of $\lambda_{a} / 2$-thick dielectric layers has already been proposed for cavity haloscopes [23] and dish antennas [26], our disks do not have to be $\lambda_{a} / 2$ thick because the coherence of the emitted waves can be controlled by the disk separations. Dielectric haloscopes can thus scan over a wide $m_{a}$ range without needing to use disks with different thicknesses for each measurement.

Axion-induced E field.-The axion-photon interaction is described by the Lagrangian density

$$
\mathcal{L}_{\text {int }}=-\frac{\alpha}{2 \pi} C_{a \gamma} \mathbf{E} \cdot \mathbf{B} \theta
$$

where $\mathbf{E}$, and $\mathbf{B}$ are electric and magnetic fields, $\theta=a / f_{a}$ represents the axion field $a$, and $C_{a \gamma}=\mathcal{E} / \mathcal{N}-1.92$ is an unknown parameter specific to each axion model, where $\mathcal{E}$ and $\mathcal{N}$ are the respective EM and color anomalies of the PQ symmetry [2]. We are interested in galactic DM axions, which are in a highly occupied state and are very nonrelativistic $\left(v_{a} \lesssim 10^{-3}\right)$. The associated de Broglie wavelength, $2 \pi / m_{a} v_{a} \gtrsim 12.4 \mathrm{~m}\left(100 \mu \mathrm{eV} / m_{a}\right)$, is larger than the dimensions of our dielectric haloscope, a cubic meter. Thus, we treat $\theta$ as a classical field and take the zerovelocity limit which implies an (approximately) homogeneous and monochromatic $\theta(t) \simeq \theta_{0} \cos \left(m_{a} t\right)$. The axion mass $m_{a}=57.0 \mu \mathrm{eV}\left(10^{11} \mathrm{GeV} / f_{a}\right)$ is related to the axion decay constant $f_{a}$ [27], which we treat as a free parameter.

The interaction (1) enters as a current on the right-hand side (rhs) of the Ampère-Maxwell equation,

$$
\nabla \times \mathbf{B}-\epsilon \dot{\mathbf{E}}=\frac{\alpha}{2 \pi} C_{a \gamma} \mathbf{B} \dot{\theta},
$$

for a medium with the permeability $\mu=1$ and a dielectric constant $\epsilon$. When a static and homogeneous external magnetic field $\mathbf{B}_{e}$ is applied, the axion DM field sources a tiny electric field

$$
\mathbf{E}_{a}(t)=-\frac{\alpha}{2 \pi \epsilon} C_{a \gamma} \mathbf{B}_{e} \theta(t),
$$

which is discontinuous at the interface between media of different $\epsilon$ 's. To satisfy the usual continuity requirements, $\mathbf{E}_{\|, 1}=\mathbf{E}_{\|, 2}$ and $\mathbf{B}_{\|, 1}=\mathbf{B}_{\|, 2}$ (recall that $\mu=1$ ), EM waves of frequency $\nu_{a}=m_{a} / 2 \pi$ must be present to compensate for the discontinuity. In effect, breaking translational invariance couples the approximately nonpropagating axion-induced $E$ field with propagating EM waves, which we aim to detect. The EM waves are emitted perpendicularly to the disk surfaces in our zero-velocity limit [25]. We require flat disks with large areas to avoid diffraction, $A \gg \lambda_{a}^{2}$, so we can work in a one-dimensional (1D) framework. For maximum effect, $\mathbf{E}_{a}$-and thus $\mathbf{B}_{e}$-must be parallel to the disk surfaces [25].

The gain from a dielectric haloscope with respect to a mirror alone can be quantified in terms of a boost factor $\beta$. Comparing the $E$ field inside the mirror $(E \simeq 0)$ to the axion-induced one in vacuum $E_{0} \equiv \alpha /(2 \pi)\left|C_{a \gamma} \mathbf{B}_{e} \theta_{0}\right|$, one finds that the continuity at the surface requires the emission of an EM wave of amplitude $E_{0}$ with a power per unit area $P_{0} / A=E_{0}^{2} / 2$ [25]. With the additional dielectric disks shown in Fig. 1, the axion field induces plane EM waves for every change of media. By tuning the disk separations, constructive interference can enhance the amplitude of the emitted EM wave to $E_{\text {out }}$ at the receiver. The boost factor is thus defined as

$$
\beta\left(\nu_{a}\right) \equiv\left|E_{\text {out }}\left(\nu_{a}\right) / E_{0}\right| .
$$

With this definition, and $E_{0}$ as introduced above, the resulting output power of the dielectric haloscope per unit area is

$$
\frac{P}{A}=\beta^{2} \frac{P_{0}}{A}=2.2 \times 10^{-27} \frac{\mathrm{W}}{\mathrm{m}^{2}} \beta^{2}\left(\frac{B_{e}}{10 \mathrm{~T}}\right)^{2} C_{a \gamma}^{2} .
$$

The boost factor is calculated by matching $\mathbf{E}_{a}$ in each region (dielectric disk or vacuum) with left- and rightmoving EM waves as imposed by the continuity of the total $\mathbf{E}_{\|}$and $\mathbf{B}_{\|}$fields at the interfaces. A transfer matrix formalism has been developed for this task [28].

The desired enhancement, $\beta \gg 1$, comes from two effects, which generally act together but can be differentiated in limiting cases. These effects depend on the optical thickness of one disk $\delta=2 \pi \nu d \sqrt{\epsilon}$, with $d$ being the physical thickness and $\nu$ the frequency, which sets the 
transmission coefficient of a single disk, found to be $\mathcal{T}=i 2 \sqrt{\epsilon} /[i 2 \sqrt{\epsilon} \cos \delta+(\epsilon+1) \sin \delta]$. When $\delta=\pi, 3 \pi$, $5 \pi, \ldots$, the disk is transparent $(\mathcal{T}=0)$ and the emission from different disks can be added constructively by placing them at the right distance. When $\mathcal{T} \neq 0$, the spacings can be adjusted to form a series of leaky resonant cavities where $E$ fields are boosted by reflections between the disks. In general, both the simple sum of the emitted waves and the resonant enhancements are important.

Comparison with cavity haloscopes.-More insight is gained by contrasting dielectric haloscopes with resonant cavities. The signal power extracted from a cavity haloscope resonantly excited by axion DM is usually expressed as [29]

$$
P_{\mathrm{cav}}=\kappa \mathcal{G} V \frac{Q}{m_{a}} \rho_{a} g_{a \gamma}^{2} B_{e}^{2},
$$

where $V$ is the cavity volume, $g_{a \gamma}=-\alpha /\left(2 \pi f_{a}\right) C_{a \gamma}, Q$ is the loaded quality factor, and $\kappa$ is the ratio of signal power to total power loss. The geometry factor $\mathcal{G}=$ $\left(\int d V \mathbf{E}_{\mathrm{cav}} \cdot \mathbf{B}_{e}\right)^{2} /\left(V B_{e}^{2} \int d V \mathbf{E}_{\mathrm{cav}}^{2}\right)$ is the normalized overlap integral of $B_{e}$ with the $E$ field of the resonant mode, $\mathbf{E}_{\text {cav }}(\mathbf{x})$, which is calculated by imposing closed boundary conditions.

By contrast, dielectric haloscopes are open systems, which are, in general, nonresonant. Nevertheless, after lengthy algebraic manipulations, one can express the boost factor (4) as an overlap integral [28],

$$
\beta \simeq \frac{m_{a}}{2 B_{e} E_{0}}\left|\int d x E_{\mathrm{dh}} B_{e}\right| .
$$

With this expression, Eq. (5) becomes highly reminiscent of Eq. (6). This can actually be turned into a dictionary: the power output of a dielectric haloscope can be calculated with Eq. (6) by defining

$$
Q_{\mathrm{dh}} \equiv \frac{1}{4} \frac{\int d x\left|E_{\mathrm{dh}}\right|^{2}}{E_{0}^{2} / m_{a}}, \quad \mathcal{G}_{\mathrm{dh}} \equiv \frac{\left|\int d x E_{\mathrm{dh}} B_{e}\right|^{2}}{L B_{e}^{2} \int d x\left|E_{\mathrm{dh}}\right|^{2}},
$$

where $L$ is the length of the haloscope. Note that $\kappa$ is implicitly contained in $\mathcal{G}_{\mathrm{dh}}$, and $Q_{\mathrm{dh}}$. Unlike for a cavity, $E_{\mathrm{dh}}(x)$ is defined as the $E$ field inside the dielectric haloscope obtained by shining in an EM wave of amplitude $E_{0}$ from the rhs in Fig. 1. This is a nontrivial conceptual change, as the physical axion-induced $E$ field (obtained with the transfer matrix formalism) is, in general, different from $E_{\mathrm{dh}}(x)$ [28]. However, when we consider highly resonant dielectric haloscopes (for instance, by choosing a very reflective rightmost disk in Fig. 1), the definitions of $E_{\text {cav }}$ and $E_{\mathrm{dh}}$ coincide as expected.

Equation (8) suggests the interpretation of $\beta^{2}$ as $m_{a} L \mathcal{G}_{\mathrm{dh}} Q_{\mathrm{dh}}$. Our proposal is to enhance $P$ by increasing $V_{\mathrm{dh}} \equiv L A, \mathcal{G}_{\mathrm{dh}}$, and $Q_{\mathrm{dh}}$ with appropriately placed disks.
This strategy sounds similar to enhancing $V$ and $\mathcal{G}$ of a strictly resonant cavity by either modifying the magnetic field [22] or using dielectrics [23,30]. However, our dielectric haloscope would be a poor resonator, compensating for a relatively low $Q_{\mathrm{dh}}$ with a huge $V_{\mathrm{dh}}$. This has completely different engineering challenges than an intermediate volume, high- $Q$ cavity.

Large- $V$ resonators are typically more complicated mechanically, which implies longer tuning times. For high- $Q$ cavities, as $V$ increases, modes tend to clutter [31], and it becomes increasingly difficult to identify and tune to them. Mode crossings become more frequent, with concomitant forbidden frequencies. Furthermore, for a large- $V$ resonant cavity, the coupling of the output port needs to increase locally to compensate for a longer time of flight of photons in the cavity, leading to stronger mode distortions.

In our case, we have the flexibility to compensate for longer tuning times by using broadband and nonresonant configurations not possible with cavity haloscopes. Furthermore, we avoid mode crossings by not having resonant modes, but rather quasimodes with very broad widths. Lastly, by making the system as $1 \mathrm{D}$ as possible with a large $A$ and extracting the signal homogeneously across the last dielectric disk before the receiver, we minimize any distortion caused by a local coupling to the detector. This allows us to avoid some of the issues of large- $V$ resonators.

Properties of the boost factor.-After choosing $\epsilon$ and the thickness of the disks $d$, the distances between disks remain as the only free parameters of our dielectric haloscope, still leaving considerable control over the frequency response. We will actually be interested in two types of configurations: one with a flat broadband response to measure a large frequency range in one go, and another with a larger $\beta$ over a narrow band to discard statistical fluctuations and do precision axion physics in case of a discovery. We can numerically generate such configurations.

One can predict the general behavior of $\beta$ by using what we call the area law: $\int \beta^{2} d \nu_{a}$ is proportional to the sum over interfaces, which holds exactly when integrating over $0 \leq \nu_{a} \leq \infty$, and is a good approximation for frequency ranges containing the main peak [28]. According to the area law, an increase in the number of disks gives a linear increase in $\int \beta^{2} d \nu_{a}$. For a single set of disks, $\int \beta^{2} d \nu_{a}$ is constant; one can trade width for power and vice versa but cannot gain in both simultaneously.

Figure 2 depicts $\beta\left(\nu_{a}\right)$ for a dielectric haloscope consisting of a mirror and 20 disks $(d=1 \mathrm{~mm}, \epsilon=25)$. Spacings have been selected to maximize the minimum boost factor $\beta_{\min }$ within three bandwidths, $\Delta \nu_{\beta}=1,50$, and $200 \mathrm{MHz}$, centered on $25 \mathrm{GHz}$ (our benchmark frequency corresponding to $\left.m_{a}=103.1 \mu \mathrm{eV}\right)$. These curves illustrate the area law: as we vary the bandwidth, the power changes by roughly the same factor. While the area is conserved, it appears to be more effectively used in 


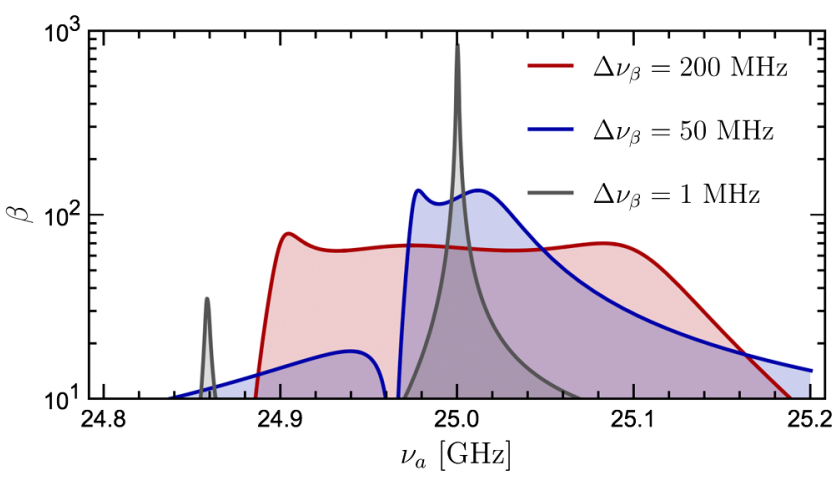

FIG. 2. Boost factor $\beta\left(\nu_{a}\right)$ for configurations optimized for $\Delta \nu_{\beta}=200,50$, and $1 \mathrm{MHz}$ (red, blue, and gray) centered on $25 \mathrm{GHz}$ using a mirror and 20 dielectric disks $(d=1 \mathrm{~mm}$, $\epsilon=25)$.

broadband configurations where we were able to obtain flatter $\beta\left(\nu_{a}\right)$ curves. Using the area law, we extrapolate to the 80 disk setup described below, reaching $\beta_{\min } \sim 275$ across $50 \mathrm{MHz}$. The achievable $\beta_{\min }$ changes with the optical thickness of a disk-in the example shown, $\delta \sim 0.8 \pi$, neither transparent nor fully reflective. Note that, for a given $d$, there are frequency bands around $\nu_{a}=$ $1,3,5, \ldots \times \pi /(\sqrt{\epsilon} d)$ for which the disks are transparent and $\beta$ is limited to the sum of the EM waves $\beta_{\min } \leq 2 N+1$, where $N$ is the number of disks. Furthermore, at $\nu_{a}=2,4,6, \ldots \times \pi / \sqrt{\epsilon} d$, the disks do not emit any radiation. Thus, when a large $\beta$ or flexibility is required, one must avoid these frequencies with a different set of disks.

Setup.-Dielectrics with a large $\epsilon$ enhance emission and resonance effects, leading to a higher $\beta$. A good candidate could be $\mathrm{LaAlO}_{3}(\epsilon \sim 25, \mu \sim 1)$, which has a very small loss tangent $\sim 10^{-5}$ at temperatures below $\sim 80 \mathrm{~K}$. The transverse area is limited by the feasibility and cost of an intense magnet: apertures larger than one square meter are extremely challenging. Dielectric disks of that area would be made by tiling smaller pieces and could be repositioned using precision motors. The precision required can be estimated by studying the analytically tractable casesthe single cavity and the $\lambda_{a} / 2$ symmetric case [26]. We find that the positioning errors have to be kept below $\sim 200 \mu \mathrm{m} \sqrt{10^{2} / \beta}\left(100 \mu \mathrm{eV} / m_{a}\right)$. For example, for $\beta \sim 10^{3}, \sigma \ll 60 \mu \mathrm{m}$ would be needed for $m_{a}=$ $100 \mu \mathrm{eV}$, though the exact sensitivity depends on the configuration. Thus, it will probably be more practical to use a broadband search technique, scanning a larger mass range in each longer measurement. We are investigating this requirement with a prototype setup using $20 \mathrm{~cm}$ diameter $\mathrm{Al}_{2} \mathrm{O}_{3}$ disks. Comparisons of the simulated and measured transmissivity and reflectivity (which are correlated with $\beta$ ) indicate that few micrometer precision could be achieved. Diffraction appears to be negligible for the setup, but full numerical studies are required.

Thermal emission of the disks and mirror contributes to the noise of the experiment, but it is suppressed when compared to a black body by both small dielectric losses and a good reflectivity, respectively. However, the haloscope will reflect thermal emission from the detector into itself. This effect can be reduced by cooling the detector to cryogenic temperatures. As a detector, we would use a broadband corrugated horn coupled to a linear amplifier like a state-of-the-art high-electron-mobility transistor (HEMT) for its broadband capabilities (operable up to $40 \mathrm{GHz}$ ) and/or quantum limited amplifiers. Using a HEMT detector at room temperature, we measured a $10^{-21} \mathrm{~W}$ signal at $17 \mathrm{GHz}$ at $6 \sigma$ in a one week run-we expect 100 times better performance at cryogenic temperatures. With $\beta \sim 400$, we would be sensitive to QCD axions.

Discovery potential.-For a practical experiment, one must scan across $m_{a}$. The procedure consists of arranging the disks to achieve a roughly constant $\beta$ in a region $\Delta \nu_{\beta}$, measuring for some time $\Delta t$ and readjusting the distances to measure an adjacent frequency range. The required $\beta$ for a reasonably short $\Delta t$ is given by Dicke's radiometer equation for the desired signal to noise ratio, $\mathrm{S} / \mathrm{N}=\left(P / T_{\text {sys }}\right) \sqrt{\Delta t / \Delta \nu_{a}}$, where the system noise temperature is $T_{\text {sys }}$ and the axion linewidth $\Delta \nu_{a} \sim 10^{-6} \nu_{a}$. Collecting the signal with efficiency $\eta$, we get

$$
\begin{aligned}
\frac{\Delta t}{1.3 \text { days }} \sim & \left(\frac{\mathrm{S} / \mathrm{N}}{5}\right)^{2}\left(\frac{400}{\beta}\right)^{4}\left(\frac{1 \mathrm{~m}^{2}}{A}\right)^{2}\left(\frac{m_{a}}{100 \mu \mathrm{eV}}\right) \\
& \times\left(\frac{T_{\text {sys }}}{8 \mathrm{~K}}\right)^{2}\left(\frac{10 \mathrm{~T}}{B_{e}}\right)^{4}\left(\frac{0.8}{\eta}\right)^{2} C_{a \gamma}^{-4} .
\end{aligned}
$$

In a single measurement, we simultaneously search $\Delta \nu_{\beta} / \Delta \nu_{a}$ possible axion "channels." Thus, the time to scan a given frequency range scales inversely to $\beta^{4} \Delta \nu_{\beta}$. As the area law implies that $\beta^{2} \Delta \nu_{\beta}$ is approximately conserved, it appears that narrow resonant peaks are optimal, as with conventional cavity haloscopes.

However, both the required placement precision and the time $t_{R}$ needed to reposition the disks limit $\beta$. With 80 disks to be adjusted, $t_{R}$ will be non-negligible - for an optimal scanning rate, the measurement and readjustment times must be similar. The value of $\beta$ required to reach $\left|C_{a \gamma}\right| \sim 1$ increases with $m_{a}$, so the dielectric haloscope could be adjusted to scan a wider mass range simultaneously at low masses and increase $\beta$, decreasing the bandwidth as the scan proceeds to higher $m_{a}$ 's. If a potential signal is found, the dielectric haloscope could be reconfigured to enhance $\beta$ at that frequency, quickly confirming or rejecting it at high significance.

In Fig. 3 we show the discovery potential of an 80 disk experiment with a run-time of $3 \mathrm{yr}$ by extrapolating from our $25 \mathrm{GHz}$ solutions using the area law. We have used $A=1 \mathrm{~m}^{2}, \epsilon=25$, and $B_{e}=10 \mathrm{~T}$. We assume $80 \%$ detection efficiency, quantum limited detection $\left(T_{\text {sys }} \sim m_{a}\right)$ and a conservative $t_{R}=1$ day. We give two examples, reaching $\left|C_{a \gamma}\right|=1$ and 0.75 in light blue and 


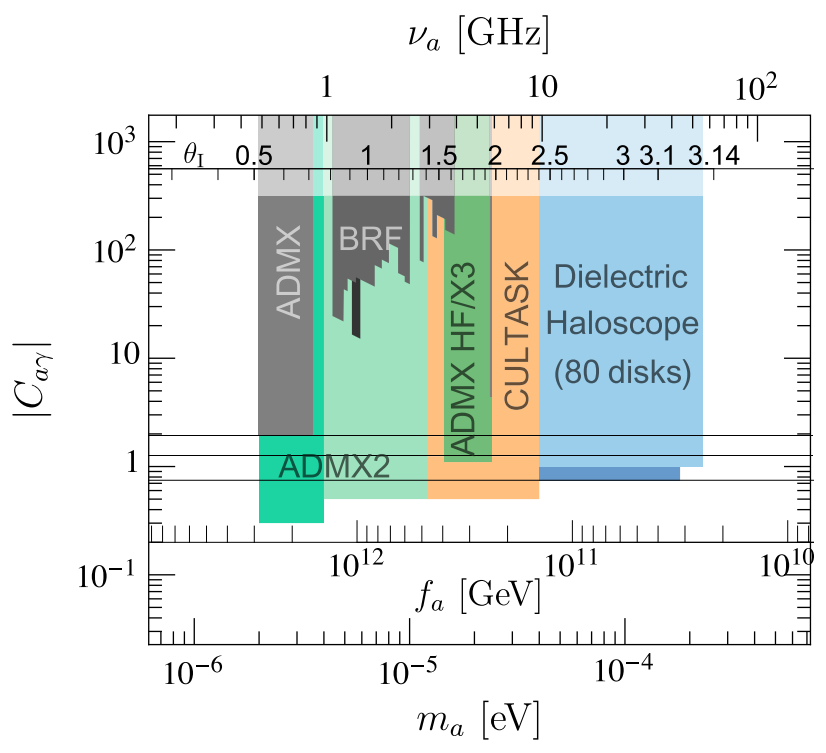

FIG. 3. Two examples of the discovery potential (light and dark blue) of our dielectric haloscope using 80 disks $(\epsilon=25$, $A=1 \mathrm{~m}^{2}, B_{e}=10 \mathrm{~T}, \eta=0.8, t_{R}=1$ day) with quantum limited detection in a 3 yr campaign. We also show exclusion limits (gray) and sensitivities (colored) of current and planned cavity haloscopes [16,17,32-36]. (Upper inset) The initial angle $\theta_{I}$ required in scenario $\mathrm{A}$ [37]. (Lower inset) The $f_{a}$ value corresponding to a given $m_{a}$. The three black lines denote $\left|C_{a \gamma}\right|=1.92,1.25,0.746$. Note that scenario B predicts $50 \mu \mathrm{eV} \lesssim m_{a} \lesssim 200 \mu \mathrm{eV}[12,38]$.

dark blue, respectively. Similar results can be achieved by using a two stage process. A 5 yr run with commercially available HEMT amplifiers with $T_{\text {sys }}=8 \mathrm{~K}$ would cover the low-mass range, for example, $m_{a} \lesssim 120 \mu \mathrm{eV}$ with $\left|C_{a \gamma}\right|=1$. The high-mass range $m_{a} \lesssim 230 \mu \mathrm{eV}$ shown in Fig. 3 would require another $2 \mathrm{yr}$ with a quantum limited detector. Adding disks and extending the run-time could expand the search to $m_{a} \lesssim 400 \mu \mathrm{eV}$.

Our haloscope would cover a large fraction of the highmass QCD axion parameter space with sensitivity to $\left|C_{a \gamma}\right| \sim 1$. In scenario A, these masses correspond to large, but still natural, initial angles $2.4 \lesssim \theta_{I} \lesssim 3.12$ [37]. Scenario B, our main goal, would be covered including the theoretical uncertainty in $m_{a}(50-200 \mu \mathrm{eV}[12,38])$ for Kim-Shifman-Vainshtein-Zakharov-type models with short-lived domain walls $(\mathcal{N}=1)$. Prime examples include the recent $\mathrm{SMASH}_{d, u}$ models $(\mathcal{E}=2 / 3,8 / 3)$ [38]. Models with $\mathcal{N}>1$ require $m_{a} \gtrsim 1 \mathrm{meV}$ [12,39], which is beyond our search range. However, with some exceptions [40], these models generally require a tuned explicit breaking of the PQ symmetry to avoid a domain-wall dominated universe [41].

Conclusion.-In this Letter we have proposed a new method to search for high-mass $(40-400 \mu \mathrm{eV})$ axions by using a mirror and multiple dielectric disks contained in a magnetic field-a dielectric haloscope. The key features are a large transverse area and the flexibility to use both broadband and narrow-band search strategies. With 80 disks, one could search a large fraction of this high-mass range with sensitivity to the QCD axion.

We acknowledge partial support by the Deutsche Forschungsgemeinschaft through Grant No. EXC 153 (Excellence Cluster "Universe") and the Alexander von Humboldt Foundation, as well as the European Commission under ERC Advanced Grant No. 339169 and under the Innovative Training Network "Elusives" Grant No. H2020MSCA-ITN-2015/674896. J. R. is supported by the Ramon y Cajal Fellowship 2012-10597 and Grant No. FPA201565745-P (MINECO/FEDER). Part of this work was performed at the Bethe Forum "Axions," Bethe Center for Theoretical Physics, University of Bonn, Germany.

[1] R. D. Peccei, The strong $C P$ problem and axions, Lect. Notes Phys. 741, 3 (2008).

[2] J. E. Kim and G. Carosi, Axions and the strong $C P$ problem, Rev. Mod. Phys. 82, 557 (2010).

[3] K. A. Olive et al. (Particle Data Group Collaboration), Review of particle physics, Chin. Phys. C 38, 090001 (2014).

[4] C. A. Baker et al., An Improved Experimental Limit on the Electric Dipole Moment of the Neutron, Phys. Rev. Lett. 97, 131801 (2006).

[5] J. Preskill, M. B. Wise, and F. Wilczek, Cosmology of the invisible axion, Phys. Lett. 120B, 127 (1983).

[6] L. F. Abbott and P. Sikivie, A cosmological bound on the invisible axion, Phys. Lett. 120B, 133 (1983).

[7] M. Dine and W. Fischler, The not so harmless axion, Phys. Lett. 120B, 137 (1983).

[8] P. Sikivie, Dark matter axions, Int. J. Mod. Phys. A 25, 554 (2010).

[9] M. Kawasaki and K. Nakayama, Axions: Theory and cosmological role, Annu. Rev. Nucl. Part. Sci. 63, 69 (2013).

[10] G. G. Raffelt, Astrophysical axion bounds, Lect. Notes Phys. 741, 51 (2008).

[11] T. Hiramatsu, M. Kawasaki, K. Saikawa, and T. Sekiguchi, Production of dark matter axions from collapse of stringwall systems, Phys. Rev. D 85, 105020 (2012); Erratum, Phys. Rev. D 86, 089902(E) (2012).

[12] M. Kawasaki, K. Saikawa, and T. Sekiguchi, Axion dark matter from topological defects, Phys. Rev. D 91, 065014 (2015).

[13] L. Fleury and G. D. Moore, Axion dark matter: Strings and their cores, J. Cosmol. Astropart. Phys. 01 (2016) 004.

[14] P. Sikivie, Experimental Tests of the Invisible Axion, Phys. Rev. Lett. 51, 1415 (1983); Phys. Rev. Lett.Erratum, 52695 (E) (1984).

[15] G. Rybka (for the ADMX Collaboration), Direct detection searches for axion dark matter, Phys. Dark Univ. 4, 14 (2014).

[16] B. M. Brubaker et al., First Results from a Microwave Cavity Axion Search at $24 \mu \mathrm{eV}$, Phys. Rev. Lett. 118, 061302 (2017).

[17] W. Chung, Launching Axion Experiment at CAPP/IBS in Korea, in Proceedings of the 12th Patras Workshop on Axions, WIMPs and WISPs, Jeju, Korea, 2016 (to be published). 
[18] D. Budker, P. W. Graham, M. Ledbetter, S. Rajendran, and A. Sushkov, Proposal for a Cosmic Axion Spin Precession Experiment (CASPEr), Phys. Rev. X 4, 021030 (2014).

[19] P. Sikivie, N. Sullivan, and D. B. Tanner, Proposal for Axion Dark Matter Detection Using an LC Circuit, Phys. Rev. Lett. 112, 131301 (2014).

[20] Y. Kahn, B. R. Safdi, and J. Thaler, Broadband and Resonant Approaches to Axion Dark Matter Detection, Phys. Rev. Lett. 117, 141801 (2016).

[21] A. Arvanitaki and A. A. Geraci, Resonantly Detecting Axion-Mediated Forces with Nuclear Magnetic Resonance, Phys. Rev. Lett. 113, 161801 (2014).

[22] G. Rybka, A. Wagner, A. Brill, K. Ramos, R. Percival, and K. Patel, Search for dark matter axions with the Orpheus experiment, Phys. Rev. D 91, 011701 (2015).

[23] D. E. Morris, Report No. LBL-17915, 1984.

[24] S. K. Lamoreaux, K. A. van Bibber, K. W. Lehnert, and G. Carosi, Analysis of single-photon and linear amplifier detectors for microwave cavity dark matter axion searches, Phys. Rev. D 88, 035020 (2013).

[25] D. Horns, J. Jaeckel, A. Lindner, A. Lobanov, J. Redondo, and A. Ringwald, Searching for WISPy cold dark matter with a dish antenna, J. Cosmol. Astropart. Phys. 04 (2013) 016.

[26] J. Jaeckel and J. Redondo, Resonant to broadband searches for cold dark matter consisting of weakly interacting slim particles, Phys. Rev. D 88, 115002 (2013).

[27] G. Grilli di Cortona, E. Hardy, J. P. Vega, and G. Villadoro, The QCD axion, precisely, J. High Energy Phys. 01 (2016) 034.

[28] A. J. Millar, G. G. Raffelt, J. Redondo, and F. D. Steffen, Dielectric haloscopes to search for axion dark matter: Theoretical foundations, J. Cosmol. Astropart. Phys. 01 (2017) 061.

[29] P. Sikivie, Detection rates for "invisible" axion searches, Phys. Rev. D 32, 2988 (1985); Erratum, Phys. Rev. D36, 974(E) (1987).

[30] G. Rybka, Commissioning of the ADMX Gen 2 Dark Matter Search, in Proceedings of the 12th Patras Workshop on Axions, WIMPs and WISPs, Jeju, Korea, 2016 (to be published).
[31] O. K. Baker, M. Betz, F. Caspers, J. Jaeckel, A. Lindner, A. Ringwald, Y. Semertzidis, P. Sikivie, and K. Zioutas, Prospects for searching axionlike particle dark matter with dipole, toroidal and wiggler magnets, Phys. Rev. D 85, 035018 (2012).

[32] S. J. Asztalos et al. (ADMX Collaboration), SQUID-Based Microwave Cavity Search for Dark-Matter Axions, Phys. Rev. Lett. 104, 041301 (2010).

[33] C. Hagmann, P. Sikivie, N. S. Sullivan, and D. B. Tanner, Results from a search for cosmic axions, Phys. Rev. D 42, 1297 (1990).

[34] W. Wuensch, S. De Panfilis-Wuensch, Y. K. Semertzidis, J. T. Rogers, A. C. Melissinos, H. J. Halama, B. E. Moskowitz, A. G. Prodell, W. B. Fowler, and F. A. Nezrick Results of a laboratory search for cosmic axions and other weakly coupled light particles, Phys. Rev. D 40, 3153 (1989).

[35] G. Carosi, Cavity-based searches for relic axions, 2016, http://bctp.uni-bonn.de/bethe-forum/2016/axions/talks/ Carosi.pdf.

[36] K. van Bibber, in Proceedings of the 11th Patras Workshop on Axions, WIMPs and WISPs, Zaragoza, Spain, 2015, edited by I. G. Irastorza, J. Redondo, J. M. Carmona, S. Cebrián, T. Dafní, F. J. Iguaz, and G. Luzón (DESY, Hamburg, 2015).

[37] S. Borsanyi et al., Calculation of the axion mass based on high-temperature lattice quantum chromodynamics, Nature (London) 539, 69 (2016).

[38] G. Ballesteros, J. Redondo, A. Ringwald, and C. Tamarit, Unifying Inflation with the Axion, Dark Matter, Baryogenesis, and the Seesaw Mechanism, Phys. Rev. Lett. 118 (2017) 071802.

[39] A. Ringwald and K. Saikawa, Axion dark matter in the post-inflationary Peccei-Quinn symmetry breaking scenario, Phys. Rev. D 93, 085031 (2016); Publisher's Note, Phys. Rev. D 94, 049908 (2016).

[40] G. Lazarides and Q. Shafi, Axion models with no domain wall problem, Phys. Lett. 115B, 21 (1982).

[41] P. Sikivie, Axions, Domain Walls and the Early Universe, Phys. Rev. Lett. 48, 1156 (1982). 